\title{
Preparation of Novel High-Temperature Polyol Esters from Vegetable Oils
}

\author{
Can Liu, ${ }^{1,2}$ Jing Liu, ${ }^{1}$ Lanqing Ma, ${ }^{2}$ and Long Rong ${ }^{1}$ \\ ${ }^{1}$ School of Biological Science and Medical Engineering, Beihang University, Room 417 Yifu Scientific Center, 37 Xueyuan Road, \\ Haidian District, Beijing 100191, China \\ ${ }^{2}$ School of Biological Science and Engineering, Beijing University of Agriculture, Beinong Road 7, Huilongguan, \\ Changping District, Beijing 102206, China
}

Correspondence should be addressed to Long Rong; ronglong64@163.com

Received 31 March 2014; Revised 23 May 2014; Accepted 25 May 2014; Published 16 June 2014

Academic Editor: Lorenzo Cerretani

Copyright (C) 2014 Can Liu et al. This is an open access article distributed under the Creative Commons Attribution License, which permits unrestricted use, distribution, and reproduction in any medium, provided the original work is properly cited.

The aim of this work was to synthesize a high-temperature polyol ester from Jatropha oil. The synthesis process was accomplished via chemical modifications involving epoxidation to remove the double bonds in Jatropha oil, hydrolysis to add hydroxyl groups, and then esterification with pentaerythritol to form the saturated polyol ester. The high decomposition temperature $359^{\circ} \mathrm{C}$ of the polyol ester was determined by thermogravimetric analysis. The lower peroxide value $0.07 \mathrm{meq} / \mathrm{kg}$ and iodine value $0.02 \mathrm{mg} \mathrm{I} / 100 \mathrm{~g}$ of the polyol esters were also determined.

\section{Introduction}

There are significant concerns regarding the current practice of using mineral oils as the primary raw materials in the lubricants industry. One major problem is that such oils, chiefly derived from petroleum distillates, are not necessarily sustainable in the long term. Furthermore, due to the inherent toxicity and nonbiodegradable nature of some mineral oil based lubricants, they may present a contamination hazard with respect to ecosystems, agricultural land, and ground water reserves [1]. As a result of these concerns, there has been increasing interest in the development of biolubricants derived from renewable resources such as vegetable oils $[2,3]$. Vegetable oils are not only biodegradable and nontoxic but also possess properties which tend to make them excellent lubricants. These characteristics include high viscosity indexes, low volatility, good lubricity, and high miscibility with other fluids $[4,5]$. However, vegetable oils typically exhibit poor thermal and oxidative stability [6]. These drawbacks presently restrict the use of vegetable oils as lubricants.

Polyol esters exhibit extraordinary stability due to the absence of hydrogen in the beta position as well as the presence of a central quaternary carbon. (1) Therefore, the thermal stability of vegetable oils can be improved by replacing the glyceride moiety in the original molecule with a polyhydric alcohol (such as trimethylolpropane or pentaerythritol) [7].

There are reports in the literature on the synthesis of polyol esters from vegetable oils $[7,8]$. However, such polyol esters retain unsaturated bonds in their fatty acid chain portions, which represent sites where the compounds may still react with atmospheric oxygen. (2) Therefore, the carbon-carbon double bond structure of the oil must be cleaved by chemical modifications to improve its thermal characteristics.

Previous research has shown that castor oil shows excellent stability due to its hydroxyl group and can form hydrogen bonding and prevent the formation of hydroperoxides [9]. It was suggested that the existence of hydroxyl groups also plays a key role in improving the thermal stability of oils. (3) Thus, attaching hydroxyl groups to the polyol ester may be another way of improving its thermal stability.

In accordance with the above-mentioned three methods of improving the stability of vegetable oil, the aim of this work was to design a high thermal polyol ester from Jatropha 


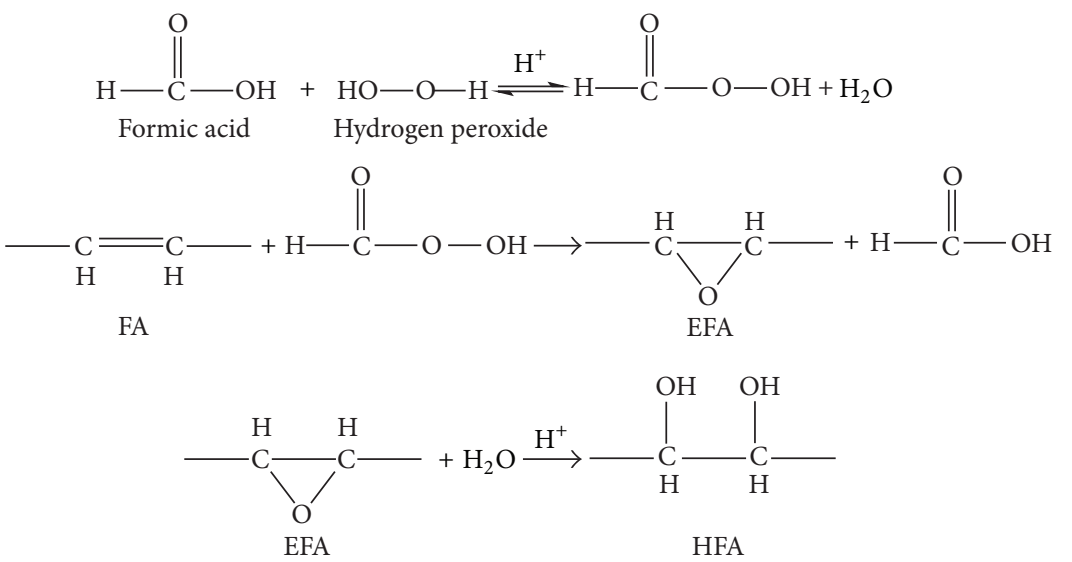

FIgUre 1: Synthetic route of HFA from FA.

oil via chemical modifications involving (1) epoxidation to remove the double bonds of Jatropha oil; (2) hydrolysis to add hydroxyl groups; (3) esterification with pentaerythritol to form saturated polyol ester. Moreover, the structure and physicochemical properties of the novel polyol ester were also determined.

\section{Materials and Methods}

2.1. Materials. Jatropha oil was obtained from Jiangsu Donghu Bio-energy Co. Ltd., Jiangsu, China. Formic acid (88\%), hydrogen peroxide (30\%), phosphotungstic acid (98\%), and pentaerythritol (98\%) were purchased from Sinopharm Chemical Reagent Co. Ltd., Shanghai, China. All the other reagents were of analytical grade.

2.2. Methods. The overall synthesis of saturated polyol esters from Jatropha oil involved three main steps. These were as follows: saponification of the oil to produce the free fatty acid, subsequent synthesis of the corresponding hydroxylated fatty acid (HFA), and, finally, esterification to give the saturated polyol ester.

2.2.1. Preparation of the Fatty Acid of Jatropha Oil. The fatty acid (FA) of Jatropha oil was prepared via a two-step process. The initial step consisted of saponification of the oil in a $\mathrm{NaOH}$ solution at $50^{\circ} \mathrm{C}$ for three hours. The resulting alkaline solution was then held at $55^{\circ} \mathrm{C}$ and neutralized by the addition of a sufficient quantity of acid, followed by washing with water. After washing, the water was removed via rotary evaporator under reduced pressure at $80^{\circ} \mathrm{C}$.

2.2.2. Preparation of the Hydroxylated Fatty Acid. Preparation of the hydroxylated fatty acid (HFA) involved sequential oxidization and hydrolysis steps [10-12]. The synthetic route of HFA was represented in Figure 1. The oxidization reaction conditions were as follows: $200 \mathrm{gFA}$ and $30 \mathrm{~g}$ formic acid (88 wt.\%) were combined in a $500 \mathrm{~mL}$ four-neck round bottom flask equipped with a thermometer, dropping funnel, water cooling condenser, and mechanical stirrer. A total of
$180 \mathrm{~g}$ of hydrogen peroxide solution (30 wt.\%) were added to the flask dropwise over $30 \mathrm{~min}$. The reaction mixture was continually stirred subsequent to the addition of the peroxide, maintaining the temperature at $50^{\circ} \mathrm{C}$, and samples were removed periodically and an iodine test for unsaturation was applied. The reaction was considered complete when the iodine test gave a value of zero, meaning all double bonds in the oil had been completely reacted. The subsequent step was hydrolysis of the epoxidized fatty acid (EFA). Water $(200 \mathrm{~mL})$ was added dropwise to the epoxide solution, following which the mixture was heated to approximately $90^{\circ} \mathrm{C}$ and stirred for five hours. This sequence of reactions very efficiently cleaves the FA double bond and attaches hydroxyl groups at the former site of unsaturation.

2.2.3. Preparation of Polyol Esters. Polyol esters were synthesized by the esterification reaction of either HFA or FA with pentaerythritol (PE) $[6,8]$. The synthesis of saturated polyol esters (SPE) was shown in Figure 2. The reaction was carried out in a three-neck, round bottom flask equipped with a Dean-Stark water separator, with constant stirring. Phosphotungstic acid was employed as a catalyst at a concentration of $2 \mathrm{wt} . \%$ (relative to the mass of the HFA). The specific reaction conditions included a temperature of $220^{\circ} \mathrm{C}$, a reaction time of 7 hours, and a molar ratio of HFA or FA : PE of $4.3: 1$. Water formed as a byproduct of the reaction was continuously removed and the quantity of captured water was used to gauge the progress of the reaction. When the reaction was complete, portions of the reaction mixture were removed, neutralized with alkaline solution, and then washed with warm $\left(60^{\circ} \mathrm{C}\right)$ water. The resulting polyol esters were dried using a rotary evaporator under reduced pressure at $85^{\circ} \mathrm{C}$. The reaction equation was as follows:

$$
4 \mathrm{HFA}+\mathrm{PE} \leftrightarrows 4 \mathrm{SEP}+4 \mathrm{H}_{2} \mathrm{O}
$$

The esterification conversion can be calculated approximately by the ratio of the amount of water actually generated to that theoretically generated. During the esterification process, the amount of fatty acids is excessive, and assuming that the $\mathrm{PE}$ reaction is completed, the mole of water theoretically 
<smiles>[R]C(O)C(O)CCCC(O)C(O)CCCCCCCC(=O)O</smiles>

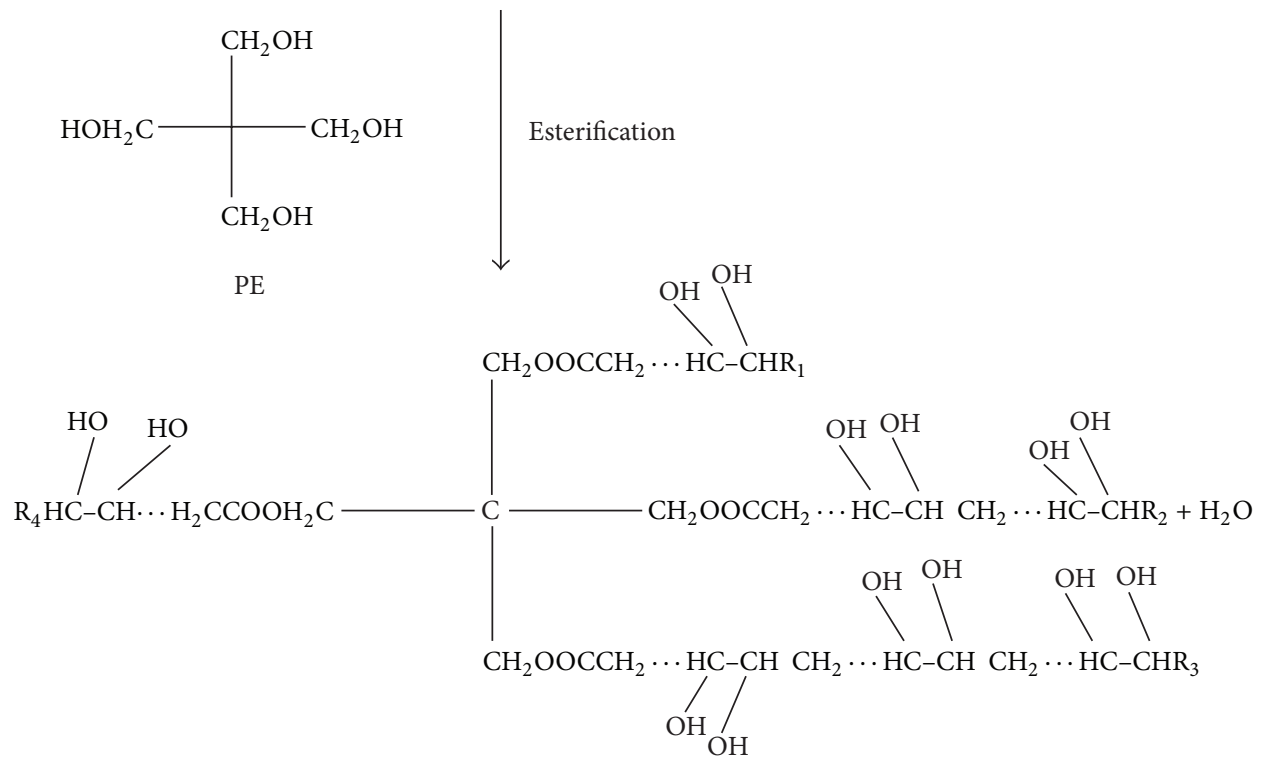

FIGURE 2: Synthesis of SEP over a phosphotungstic acid catalyst.

generated is four times the amount of PE. For example, if the amount of reactant PE is $0.2 \mathrm{~mol}$, in theory, the amount of water generated is $0.8 \mathrm{~mol}$ in this reaction. The relationship between the mole of initial PE and the mole of water theoretically generated could be expressed as follows:

$$
M_{2}=\frac{M_{1}}{4},
$$

where $M_{1}$ is the mole of the generated water and $M_{2}$ is the mole of initial PE.

The esterification conversion was calculated by the next equation as follows:

$$
C=\frac{M}{18 M_{2}} \times 100 \%=\frac{2 M_{1}}{9},
$$

where $C$ is the conversion and $M$ is the actual mass of water obtained after the esterification process.

\subsection{Physicochemical Properties Determination. Fourier} transform infrared (FTIR) spectroscopy was used for structural characterization. Spectra were recorded with a FTIR spectroscope (Gangdong 650) over $500-4000 \mathrm{~cm}^{-1}$ using 32 scans at a resolution of $4 \mathrm{~cm}^{-1}$. The thermal stability of each sample was examined using a STA 449C thermogravimetric analysis (TGA) apparatus (Netzsch, Waldkraiburg, Germany).

The iodine values of samples were determined in accordance with the ASTM D5554-95 standard method. The

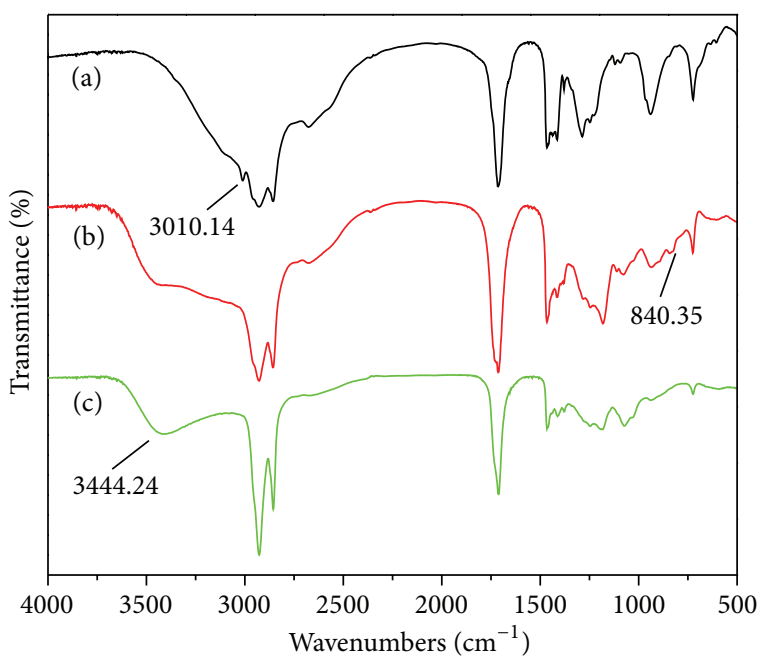

Figure 3: FTIR spectra of FA (a), EFA (b), and HFA (c).

peroxide values of samples were determined according to the AOCS Cd 8-53 standard method.

\section{Results and Discussion}

FTIR analysis was used to identify the products at different reaction stages. Figure 3 shows the spectra of the FA, EFA, 


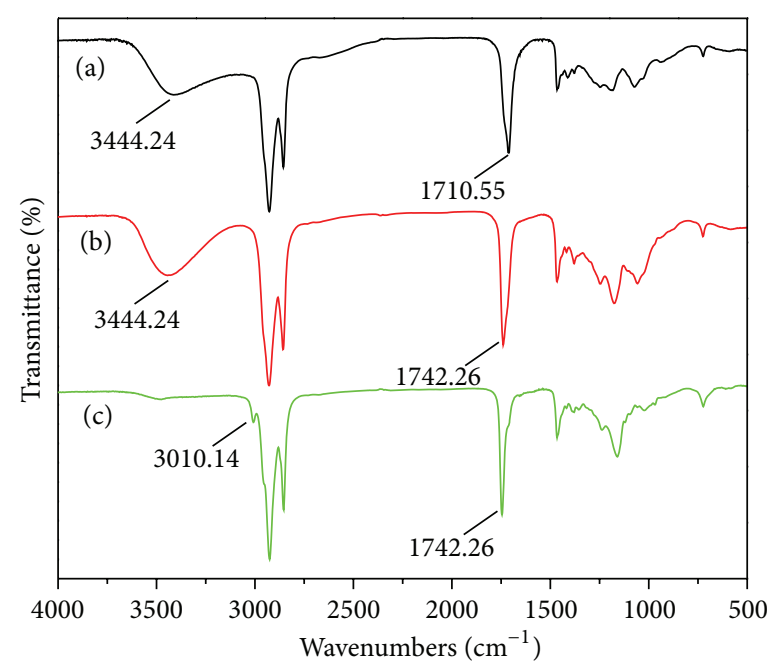

FIgURE 4: FTIR spectra of HFA (a), SPE (b), and UPE (c).

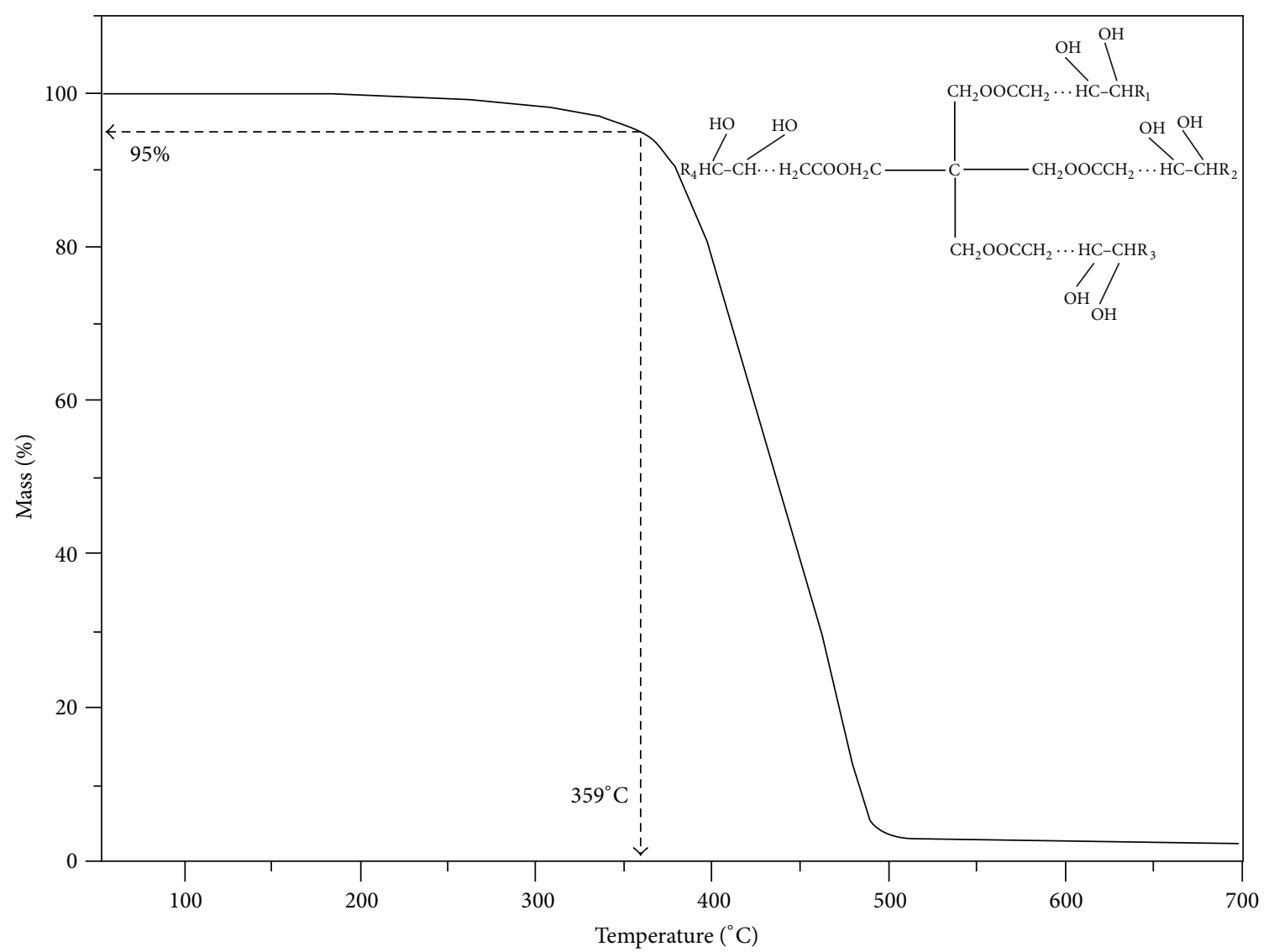

FIgURE 5: TGA thermogram of the SPE.

and HFA products. It is evident that, following the oxidization reaction, the peak at $3010 \mathrm{~cm}^{-1}$ corresponding to the double bond stretching vibration is lost, while a small peak at $840 \mathrm{~cm}^{-1}$ due to the epoxide group appears [13]. This confirms the reaction of the double bond to the epoxide.
As the subsequent hydroxylation reaction progressed, it was observed that the epoxide peak gradually diminished while a hydroxyl band at approximately $3444 \mathrm{~cm}^{-1}$ became increasingly prominent [14]. At the completion of the reaction, the complete disappearance of both the double bond 
TABLE 1: Iodine and peroxide test data for Jatropha oil, UPE, and SPE.

\begin{tabular}{lccc}
\hline Parameter & Jatropha oil & UPE & SPE \\
\hline Iodine value $\left(\mathrm{mg} \mathrm{I}_{2} / 100 \mathrm{~g}\right)$ & 100.52 & 89.43 & 0.02 \\
Peroxide value $(\mathrm{meq} / \mathrm{kg})$ & 16.39 & 23.52 & 0.07 \\
\hline
\end{tabular}

$\left(3010 \mathrm{~cm}^{-1}\right)$ and epoxide peaks $\left(840 \mathrm{~cm}^{-1}\right)$ indicated that all of the double bonds were successfully converted via the addition of hydroxyl groups.

The synthesis of SPE was accomplished by an esterification reaction between HFA and PE, and 93.5\% conversion was obtained. Initially, unsaturated polyol esters (UPE) were synthesized by the esterification of FA with PE. As shown in Figure 4, after esterification, there is a shift in the peak at $1710 \mathrm{~cm}^{-1}$, which is characteristic of FAs, to $1742 \mathrm{~cm}^{-1}$ which corresponds to the ester linkage, confirming the formation of ester groups. Comparing the spectra of SPE with UPE, it is evident that there are significant differences in the peaks corresponding to both the hydroxyl group and the double bond. As expected, the SPE is characterized by a broad hydroxyl stretching peak around $3444 \mathrm{~cm}^{-1}$ and the complete disappearance of the double bond peak at $3010 \mathrm{~cm}^{-1}$, while the UPE exhibits a peak for the double bond but not the hydroxyl group.

The weight loss (TGA) curve acquired under a helium atmosphere for the saturated polyol ester (SPE) is presented in Figure 5. The starting temperature for thermal decomposition is taken as the temperature at which samples showed a five-percent weight loss. It can be seen that the SPE displayed excellent thermal stability and began to degrade at $359^{\circ} \mathrm{C}$. The high decomposition temperature of the SPE can be explained by the following reasons: (1) the SPE has no hydrogen in the beta position [8]; (2) the SPE has hydroxyl groups and hence additional hydrogen bonding [15]; (3) the SPE has no unsaturated bonds.

Table 1 provides data concerning the values of iodine and peroxide tests for both the UPE and SPE, as well as for unmodified Jatropha oil. Since the iodine value is a measure of the unsaturation of fats and oils, the SPE has the lowest value. The peroxide value of an oil is considered one of its most important parameters, as it measures the total amount of peroxide in the oil and hence the extent to which it has undergone oxidation. As such, since oxidative stability of oils can be defined as resistance to oxidation, a low peroxide value for lubricants corresponds to better oxidative resistance. Table 1 shows that the peroxide value of SPE is much lower than that of either pure Jatropha oil or the UPE, since the SPE is saturated while the others both have a significant number of unsaturated sites which are very sensitive to autoxidation and can be rapidly transformed into peroxides $[16,17]$. These results confirm that the SPE has much better oxidative stability. Vegetable oil based lubricants, however, typically exhibit poor thermal and oxidative stability, since the beta hydrogen on the glyceride moiety is readily eliminated, leading to subsequent cleavage of the ester into the corresponding acid and olefin. This work was attempted to improve both the thermal and oxidative stability of Jatropha vegetable oil. This was accomplished via a series of chemical modifications involving epoxidation, hydroxylation, and finally esterification with pentaerythritol. This sequence of reactions works efficiently to add hydroxyl groups across the double bond within the Jatropha oil fatty acid as well as to eliminate the beta hydrogen on the glyceride portion of the molecule. Compared to UPE (unsaturated polyol esters), SPE (saturated polyol ester) shows much lower iodine value and peroxide value.

\section{Conclusions}

In this work, a novel high-temperature polyol ester from Jatropha oil was successfully synthesized. The high decomposition temperature $359^{\circ} \mathrm{C}$ of the polyol ester was determined by thermogravimetric analysis. Compared with the raw material Jatropha oil, the lower peroxide value $0.07 \mathrm{meq} / \mathrm{kg}$ and iodine value $0.02 \mathrm{mg} \mathrm{I}_{2} / 100 \mathrm{~g}$ of the polyol esters were also obtained. Considering its high thermal stability, the polyol ester may provide the conditions and potential for the development of new high-temperature lubricants.

\section{Conflict of Interests}

The authors declare that there is no conflict of interests regarding the publication of this paper.

\section{Acknowledgment}

This work was supported by foundation of "Da Bei Nong" no. $13 \mathrm{Z}$ K001.

\section{References}

[1] D. R. Kodali, "High performance ester lubricants from natural oils," Industrial Lubrication and Tribology, vol. 54, no. 4, pp. 165170, 2002.

[2] S. Z. Erhan and S. Asadauskas, "Lubricant basestocks from vegetable oils," Industrial Crops and Products, vol. 11, no. 2-3, pp. $277-282,2000$

[3] H. Wagner, R. Luther, and T. Mang, "Lubricant base fluids based on renewable raw materials: their catalytic manufacture and modification," Applied Catalysis A: General, vol. 221, no. 1-2, pp. 429-442, 2001.

[4] H.-S. Hwang and S. Z. Erhan, "Synthetic lubricant basestocks from epoxidized soybean oil and Guerbet alcohols," Industrial Crops and Products, vol. 23, no. 3, pp. 311-317, 2006.

[5] R. N. M. Kamil and S. Yusup, "Modeling of reaction kinetics for transesterification of palm-based methyl esters with trimethylolpropane," Bioresource Technology, vol. 101, no. 15, pp. 5877$5884,2010$.

[6] N. H. Arbain and J. Salimon, "Synthesis and characterization of ester trimethylolpropane based Jatropha Curcas oil as biolubricant base stocks," Journal of Science Technology, vol. 2, no. 2, pp. 47-58, 2011.

[7] R. N. M. Kamil, S. Yusup, and U. Rashid, "Optimization of polyol ester production by transesterification of Jatropha-based methyl ester with trimethylolpropane using Taguchi design of experiment," Fuel, vol. 90, no. 6, pp. 2343-2345, 2011. 
[8] S. Gryglewicz, W. Piechocki, and G. Gryglewicz, "Preparation of polyol esters based on vegetable and animal fats," Bioresource Technology, vol. 87, no. 1, pp. 35-39, 2003.

[9] D. Ogunniyi, "Castor oil: a vital industrial raw material," Bioresource Technology, vol. 97, no. 9, pp. 1086-1091, 2006.

[10] P. Tran, D. Graiver, and R. Narayan, "Ozone-mediated polyol synthesis from soybean oil," Journal of the American Oil Chemists Society, vol. 82, no. 9, pp. 653-659, 2005.

[11] P. Meyer, N. Techaphattana, S. Manundawee, S. Sangkeaw, W. Junlakan, and C. Tongurai, "Epoxidation of soybean oil and Jatropha oil," Thammasat International Journal of Science and Technology, vol. 13, pp. 1-5, 2008.

[12] A. Adhvaryu and S. Erhan, "Epoxidized soybean oil as a potential source of high-temperature lubricants," Industrial Crops and Products, vol. 15, no. 3, pp. 247-254, 2002.

[13] L. K. Jia, L. X. Gong, W. J. Ji, and C. Y. Kan, "Synthesis of vegetable oil based polyol with cottonseed oil and sorbitol derived from natural source," Chinese Chemical Letters, vol. 22, no. 11, pp. 1289-1292, 2011.

[14] R. Briones, L. Serrano, R. Llano-Ponte, and J. Labidi, "Polyols obtained from solvolysis liquefaction of biodiesel production solid residues," Chemical Engineering Journal, vol. 175, no. 1, pp. 169-175, 2011.

[15] Z. S. Petrovic, W. Zhang, and I. Javni, "Structure and properties of polyurethanes prepared from triglyceride polyols by ozonolysis," Biomacromolecules, vol. 6, no. 2, pp. 713-719, 2005.

[16] H. Azeredo, J. A. F. Faria, and M. A. A. P. da Silva, "Minimization of peroxide formation rate in soybean oil by antioxidant combinations," Food Research International, vol. 37, no. 7, pp. 689-694, 2004.

[17] A. Dhaouadi, L. Monser, S. Sadok, and N. Adhoum, "Flowinjection methylene blue-based spectrophotometric method for the determination of peroxide values in edible oils," Analytica Chimica Acta, vol. 576, no. 2, pp. 270-274, 2006. 

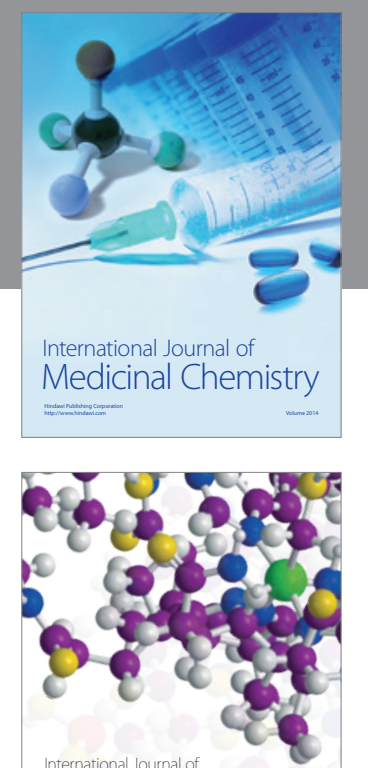

\section{Carbohydrate} Chemistry

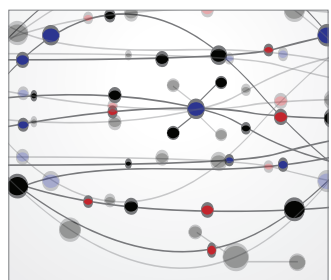

The Scientific World Journal
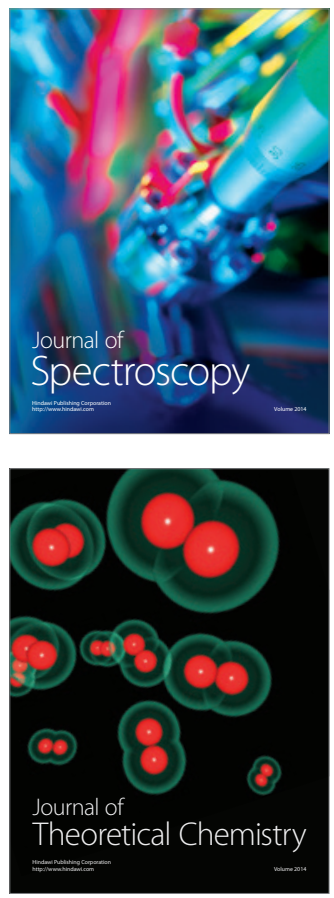
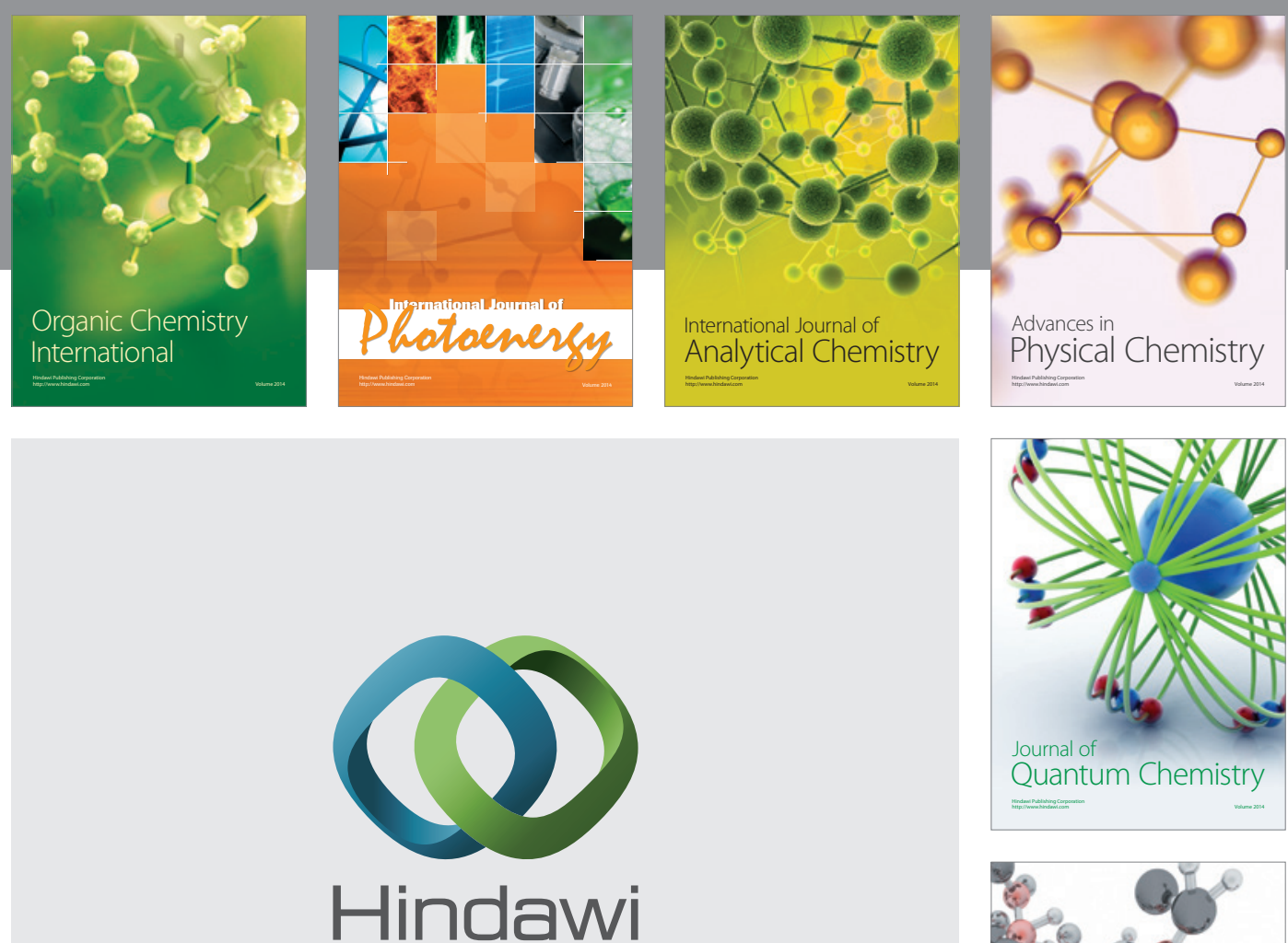

Submit your manuscripts at

http://www.hindawi.com

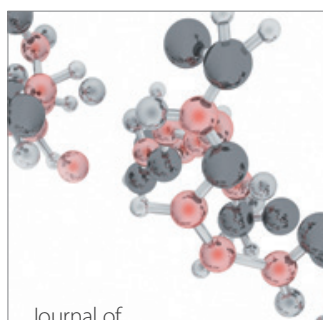

Analytical Methods

in Chemistry

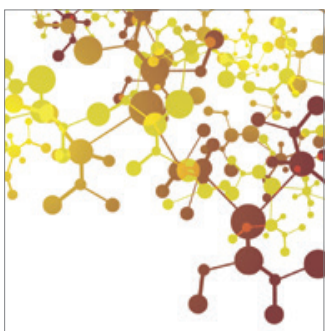

Journal of

Applied Chemistry

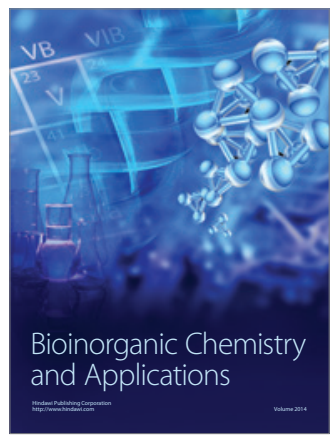

Inorganic Chemistry
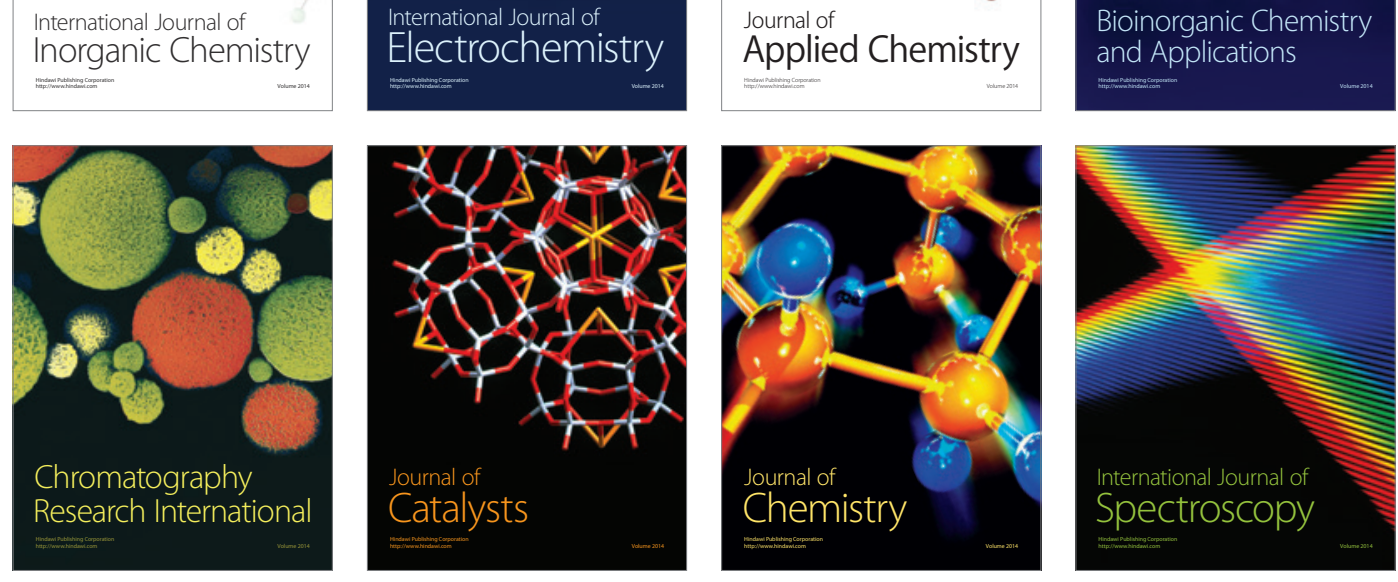